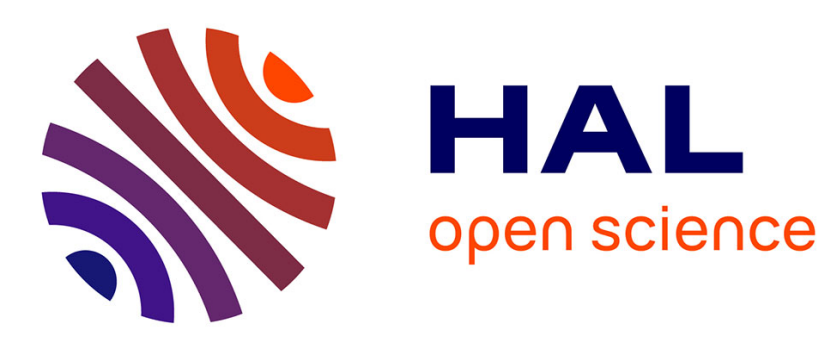

\title{
A spillover phenomenon in the optimal location of actuators
}

\author{
Pascal Hebrard, Antoine Henrot
}

\section{To cite this version:}

Pascal Hebrard, Antoine Henrot. A spillover phenomenon in the optimal location of actuators. SIAM Journal on Control and Optimization, 2005, 44 (1), pp.349-366. 10.1137/S0363012903436247 . hal00013870

\section{HAL Id: hal-00013870 https://hal.science/hal-00013870}

Submitted on 15 Nov 2005

HAL is a multi-disciplinary open access archive for the deposit and dissemination of scientific research documents, whether they are published or not. The documents may come from teaching and research institutions in France or abroad, or from public or private research centers.
L'archive ouverte pluridisciplinaire HAL, est destinée au dépôt et à la diffusion de documents scientifiques de niveau recherche, publiés ou non, émanant des établissements d'enseignement et de recherche français ou étrangers, des laboratoires publics ou privés. 


\title{
A SPILLOVER PHENOMENON IN THE OPTIMAL LOCATION OF ACTUATORS*
}

\author{
PASCAL HÉBRARD ${ }^{\dagger}$ AND ANTOINE HENROT ${ }^{\ddagger}$
}

\begin{abstract}
In this paper, we are interested in finding the optimal location and shape of the actuators in a stabilization problem. Namely, we consider the one-dimensional wave equation damped by an internal feedback supported on a subdomain $\omega$ of given length. The criterion we want to optimize represents the rate of decay of the total energy of the system. It theoretically involves all the eigenmodes of the operator. From an engineering point of view, it seems more realistic to consider only a finite number of modes, say the $N$ first ones. In that context, we are able to prove existence and uniqueness of an optimal domain $\omega_{N}^{*}$ : it is the better possible location for the actuators. We characterize this optimal domain and we point out the following strange phenomenon (at least for small lengths): the optimal domain $\omega_{N}^{*}$ which is the better one for the $N$ first modes is actually the worse one for the $N+1$-th mode. This looks like to the well-known spillover phenomenon in Control Theory. At last, we will give some possible extension and open problems in higher dimension.
\end{abstract}

Key words. damped wave equation, optimal location, spillover, stabilization

AMS subject classifications. 49J20,93B55,93C20,35L05

1. Introduction. In control and stabilization problems, the choice of the best location (and shape) of the actuators is a very important and practical question. Among criterion which can be studied, the rate of decay of the energy of the system is an important one since it does not depend on the initial conditions. In the onedimensional problem that we are going to consider here (wave equation with internal distributed control), it is known that this rate of decay is precisely the opposite of the spectral abscissa of the corresponding operator (see [9]). Therefore, optimizing this rate of decay consists in pushing all the eigenvalues as far as possible to the left in the complex plane. Among works in this direction, we refer e.g. to [8] where is proved that a constant damping is a local maximizer of the rate of decay, [11] which shows that the constant damping is not a global maximizer and [5] where they show that we can achieve an arbitrarily large rate of decay by considering damping of the kind $a(x)=1 /(x+b)$ (see below for the mathematical model). In this work, we will restrict ourselves to a damping of the kind $k \chi_{\omega}(x)$ where $k$ is a (small) positive constant and $\chi_{\omega}$ is the characteristic function of a sub-domain $\omega$ of the string which is our main unknown.

In higher dimension, this spectral abscissa is also an important component of the rate of decay, but we must also consider a geometric quantity describing the time each high frequency (or waves with a little wave length) stays in the zone of control, see [3], [18].

From an engineering point of view, it seems to be difficult (and perhaps useless) to take into account an infinite number of modes. So, a more reasonable version of this problem would be to consider only the $N$ first modes. Indeed, the high frequencies are not too much penalizing for the vibrating structure. The aim of this paper is to show that if we choose the optimal domain, say $\omega_{N}^{*}$, for the $N$ first modes (we will prove that it exists and it is unique), this domain behaves very badly for the first mode that we have forgotten: the $(N+1)$-th mode! More precisely, $\omega_{N}^{*}$ generally

\footnotetext{
*THIS PAPER IS ACCEPTED IN SIAM JOURNAL OF CONTROL AND OPTIMIZATION

†Institut Élie Cartan, email:pascal_hebrard@ds-fr.com

$\ddagger$ (corresponding author), Institut Élie Cartan and École des Mines Nancy, email: henrot@iecn.unancy.fr
} 
concentrates on the nodes of the $(N+1)$-th mode, and therefore does not control it at all. Roughly speaking, the best domain for the $N$ first modes is the worse one for the $(N+1)$-th mode! In this paper, we are able to prove this result for dampers with small support, but actually we can observe numerically that, in general, the best damper for the $N$ first modes behaves very badly for the $N+1$-th mode, see Figure 5.1 and section 5.2. Since the choice of $N$ is generally arbitrary, it seems to be a very bad idea to look for the optimal zone of control for the $N$ first modes. It is, somehow, as if we push the energy after the $N$ first modes like in classical spill-over phenomena, as described for example in [2]. In our paper, we choose to consider a wave packet constituted with all the low frequencies. In some sense, the phenomenon which occurs here seems similar to the one due to different group velocity as described in [25], see also [26] for a survey, but in our case it is not only a question of low and high frequencies. Indeed, we could have chosen other wave packets, in particular with different group velocities. Actually, we can observe (at least numerically, we did not write proofs) the same phenomenon for any choice of wave packets. For example, if we want to damp at best the packet of eigenfrequencies $\lambda_{1}, \lambda_{4}, \lambda_{5}$, the optimal domain we get (which still exists and is unique) will mainly concentrate on the nodes of the second eigenfunction $\phi_{2}$, which is the first frequency we have forgotten, and therefore will be unable to damp correctly this eigenmode. Nevertheless, E. Zuazua claims in [26] controlling a discrete version of a continuous wave model is often a bad way of controlling the continuous wave model itself. We have another illustration of this phenomenon here.

The plan of this paper is the following. Section 2 deals with the mathematical model which is used and fix the notations. In section 3 we prove existence and uniqueness of the optimal domain and we characterize it. Section 4 is devoted to describe and prove the kind of spill-over phenomenon that we have just described above. At last, in section 5, we will give some remarks and possible extensions to the two-dimensional case.

2. The mathematical model. Let us now give the model and the notations that we are going to use throughout this paper. We consider a string (a one-dimensional model), but it is essentially for technical reasons. We will say a few words, in section 5, about higher dimensional models, pointing out what has to be done to generalize our results.

So, let us denote by $\Omega=(0,1)$ the unit string that we suppose fixed at its extremities. We want to stabilize this string thanks to a damping acting only on a subdomain $\omega$. More precisely, we consider the following modelling. The displacement $u$ of the string in presence of viscous damping $2 k_{\omega}$ (where $\chi_{\omega}$ denotes the characteristic function of the subdomain $\omega$ of positive length), satisfies the damped wave equation:

$$
\left\{\begin{array}{l}
u_{t t}(x, t)-u_{x x}(x, t)+2 k \chi_{\omega}(x) u_{t}(x, t)=0, x \in(0,1), t>0 \\
u(0, t)=u(1, t)=0, t>0
\end{array}\right.
$$

upon being set in motion by the initial disturbance

$$
u(x, 0)=u_{0}(x), \quad u_{t}(x, 0)=u_{1}(x), \quad \forall x \in[0,1] .
$$

The energy of the string at time $t$ is defined by:

$$
E(t)=\int_{0}^{1}\left[u_{x}^{2}(x, t)+u_{t}^{2}(x, t)\right] d x
$$


If $\omega$ has positive measure, this system is exponentially stable, i.e. its energy is known to obey (see e.g. [6], [9]) :

$$
E(t) \leq C E(0) e^{-2 \tau t}
$$

for some constants $C>0$ and $\tau>0$ independent of the initial data. We define the decay rate, as a function of $k$ and $\omega$, to be the largest such $\tau$ :

$$
\begin{array}{r}
\tau(k, \omega)=\sup \left\{\tau^{\prime}: \exists C\left(\tau^{\prime}\right)>0 \text { s.t. } E(t) \leq C E(0) e^{-2 \tau^{\prime} t},\right. \\
\text { for every solution of }(2.1) \text { and }(2.2)\}
\end{array}
$$

Cox and Zuazua have shown in [9] that if $\chi_{\omega}$ is of bounded variation i.e. $\omega$ is the union of a finite number of intervals, then $\tau(k, \omega)$ is equal to the opposite of the spectral abscissa of the operator A :

$$
\tau(k, \omega)=-\mu=-\sup \{\operatorname{Re} \lambda: \lambda \in \operatorname{sp}(A)\}
$$

where $A$ denotes the linear operator associated to equation (2.1) :

$$
A=\left(\begin{array}{cc}
0 & I \\
\frac{d^{2}}{d x^{2}} & -2 k \chi_{\omega}(x)
\end{array}\right), \quad D(A)=\left(H^{2}(0,1) \cap H_{0}^{1}(0,1)\right) \times H_{0}^{1}(0,1)
$$

and $\operatorname{sp}(A)$ its spectrum. Therefore, a natural question would be to look for $k$ and $\omega$ which minimize this spectral abscissa (or maximize $\tau(k, \omega)$ ).

In such a generality, looking for the maximizer of $(k, \omega) \mapsto \tau(k, \omega)$ is quite difficult. In [15], we explain (and we give justifications) how to simplify the problem by considering, instead of the decay rate, the quantity

$$
J(\omega)=\inf _{n \in \mathbb{N}^{*}} \int_{0}^{1} \chi_{\omega}(x) \phi_{n}^{2}(x) d x
$$

where $\mathbb{N}^{*}$ stands for the set of positive integers and $\left(\phi_{n}\right)_{n \in \mathbb{N}^{*}}$ denote the normalized eigenfunctions for the problem without damping, i.e. $\phi_{n}=\sqrt{2} \sin n \pi x$. Actually, when $k$ is not too large, we have $\tau(k, \omega) \simeq k J(\omega)$, since $J(\omega)$ is nothing else but the derivative of $\tau$ with respect to $k$ for $k=0$. On the other hand, taking $k$ large is not interesting at all, due to the classical overdamping phenomenon described e.g. in [9], [12], [15]. Therefore, $J(\omega)$ gives a good approximation of the decay rate for small $k$. We also refer to [13] for a similar analysis.

As explained in the Introduction, it seems more realistic, at least from an engineering point of view, to take into consideration only a finite number of modes. It means that it seems reasonable to replace the functional $J$ by the simpler $J_{N}$ (where $N$ is a given integer), defined by:

$$
J_{N}(\omega)=\min _{1 \leq n \leq N} \int_{0}^{1} \chi_{\omega}(x) \phi_{n}^{2}(x) d x
$$

Therefore, we are interested in solving the following problem:

$$
\mathcal{P}_{\omega} \quad\left\{\begin{array}{l}
\text { Find } \left.\omega^{*} \text { subset of }\right] 0,1[\text { of measure } l \text { which maximizes } \\
J_{N}(\omega)=\min _{1 \leq n \leq N} 2 \int_{0}^{1} \chi_{\omega}(x) \sin ^{2}(n \pi x) d x
\end{array}\right.
$$

In the sequel, for each integer $k$ and each function $a$, we will denote by $j_{k}(a)$ the quantity

$$
j_{k}(a)=\int_{0}^{1} a(x) \phi_{k}^{2}(x) d x
$$


We could also wonder whether $J_{N}$ is a "good" approximation of $J$. Since it is proved in [15] that $J$ has no maximizer in the class of characteristic functions (except for the particular case $l=0.5$ ) while $J_{N}$ has always a (unique) maximizer in this class (as proved below), it seems at first sight that it is not a good approximation. But, it becomes a good one if we accept to work in the convex set $\mathcal{A}_{l}$ defined in (3.1). Actually, we can prove:

Proposition 2.1. Let us consider the functionals $J_{N}$ and $J$ defined on the convex set $\mathcal{A}_{l}$ (defined in (3.1)) respectively by $J_{N}(a)=\min _{1 \leq n \leq N} j_{n}(a)$ and $J(a)=$ $\inf _{n \in \mathbb{N}^{*}} j_{n}(a)$.

Then $J_{N} \Gamma$-converge to $J$ in the sense of De Giorgi. Moreover, let $\chi_{\omega_{N}^{*}}$ be the sequence of maximizers of $J_{N}$ given by Theorem 3.1, then $\chi_{\omega_{N}^{*}}$ converges (up to a subsequence) weak-* to a maximizer of $J$ and $\max _{\mathcal{A}_{l}} J=\lim _{N \rightarrow+\infty} J_{N}\left(\chi_{\omega_{N}^{*}}\right)$.

Proof. Since we are interested in a maximization problem, the definition of $\Gamma$ convergence reads here, see e.g. [10]:

(i) For all sequence $a_{n}$ in $\mathcal{A}_{l}$ which converge weak-* to $a, J(a) \geq \limsup J_{N}\left(a_{N}\right)$.

(ii) There exists one sequence $a_{n}$ in $\mathcal{A}_{l}$ which converge weak-* to $a$, such that $J(a) \leq$ $\liminf J_{N}\left(a_{N}\right)$.

For (i), let us fix $\varepsilon>0$ and choose an integer $k_{0}$ such that $J(a) \leq j_{k_{0}}(a) \leq J(a)+\varepsilon$. For every integer $N \geq k_{0}$, we have

$$
J_{N}\left(a_{N}\right) \leq j_{k_{0}}\left(a_{N}\right) .
$$

Now, $j_{k_{0}}\left(a_{N}\right) \rightarrow j_{k_{0}}(a)$ when $N \rightarrow+\infty$, therefore taking the lim-sup in both sides of (2.7) yields $\limsup J_{N}\left(a_{N}\right) \leq \limsup j_{k_{0}}\left(a_{N}\right)=j_{k_{0}}(a) \leq J(a)+\varepsilon$ which gives (i) since $\varepsilon$ is arbitrary.

For (ii), it suffices to consider a constant sequence $a_{n}=a$ since $J(a) \leq J_{N}(a)$.

Now, the last two claims come directly from the classical Theorem of De Giorgi (see [10]) and the fact that the sequence $\chi_{\omega_{N}^{*}}$ is pre-compact in $\mathcal{A}_{l}$.

The functional $J$ may have several maxima but the characterization of $\omega_{N}^{*}$ which is given below (Theorem 4.1) shows that $\chi_{\omega_{N}^{*}}$ converges actually to the constant function $a(x)=l$ which is the more natural maximizer.

3. Existence, Uniqueness and Characterization of the optimum. We begin by proving the following existence and uniqueness result for the optimal domain.

THEOREM 3.1. The problem $\mathcal{P}_{\omega}$ has a unique solution $\omega_{N}^{*}$. This solution is an union of at most $N$ intervals. It is symmetric with respect to $1 / 2$.

The proof of Theorem 3.1 will be done in several steps. First of all, we are going to use some kind of relaxation of the problem by introducing the convex hull $\mathcal{A}_{l}$ of the set of characteristic functions. Existence of an optimum in this set will be obtained easily. By characterization of this optimum thanks to the optimality conditions, we will be able to prove that it is indeed an extreme point of $\mathcal{A}_{l}$, i.e. the characteristic function of a sub-domain. Uniqueness will then follow from the fact that the functional $J$ is concave.

1st step, relaxation: The maximization problem is posed on the set of characteristic functions

$$
\mathcal{L}_{l}=\left\{a(x) \in L^{\infty}(0,1), a(x)=0 \text { or } 1 \text { a.e., } \int_{0}^{1} a(x) d x=l\right\} .
$$

This set is not very convenient for this maximization problem, since it is not closed for the natural topology associated to the functional $J_{N}$, namely the weak-star topology 
on $L^{\infty}(0,1)$. Indeed $J_{N}$ is clearly continuous for this topology. So, let us introduce the convex hull of $\mathcal{L}_{l}$ :

$$
\mathcal{A}_{l}=\left\{a(x) \in L^{\infty}(0,1), 0 \leq a(x) \leq 1, \int_{0}^{1} a(x) d x=l\right\}
$$

which is also the closure of $\mathcal{L}_{l}$ for the weak-star topology on $L^{\infty}(0,1)$. The set $\mathcal{A}_{l}$ is compact for this topology, while $\mathcal{L}_{l}$ coincides with the set of extreme points of $\mathcal{A}_{l}$, see e.g. [16]. Moreover, $J_{N}$ has a natural extension (always denoted by $J_{N}$ ) to $\mathcal{A}_{l}$ defined by

$$
\forall a \in \mathcal{A}_{l}, \quad J_{N}(a)=\min _{1 \leq n \leq N} \int_{0}^{1} a(x) \phi_{n}^{2}(x) d x
$$

It is clear that $J_{N}$ is continuous on $\mathcal{A}_{l}$ for the weak-star topology. Therefore, $J_{N}$ admits (at least) a maximum in $\mathcal{A}_{l}$.

2nd step, optimality conditions: Let $a^{*}$ such a maximum, and let us denote by $I\left(a^{*}\right)$ the active index-set:

$$
I\left(a^{*}\right)=\left\{k \in\{1,2, \ldots, N\}, \text { such that } j_{k}\left(a^{*}\right)=J_{N}\left(a^{*}\right)\right\} .
$$

It is well-known in non-smooth analysis (see e.g. [17]) that the sub-differential of $J_{N}$ at $a^{*}$ is given by:

$$
\partial J_{N}\left(a^{*}\right):=\operatorname{co}\left\{\cup \partial j_{k}\left(a^{*}\right), k \in I\left(a^{*}\right)\right\}
$$

where co denotes the convex hull. Now, the $j_{k}$ being linear, they are equal to their differential and the optimality condition reads:

$$
0 \in \partial J_{N}\left(a^{*}\right)+\lambda_{0} L_{0}
$$

(where $\lambda_{0}$ stands for a Lagrange multiplier taking into account the length constraint and $L_{0}$ is the linear form defined by $\left.\left.<L_{0}, h\right\rangle=\int_{0}^{1} h(x) d x\right)$, what yields, thanks to $(3.3)$ :

$$
\left\{\begin{array}{c}
\exists\left(\lambda_{k}\right) \in[0,1], k \in I\left(a^{*}\right), \sum_{k} \lambda_{k}=1, \exists \lambda_{0} \in \mathbb{R} \text { such that } \\
\forall h \in L^{\infty}([0,1]), h \text { admissible }, \\
\sum_{k \in I\left(a^{*}\right)} \lambda_{k} \int_{0}^{1} h(x) \phi_{k}^{2}(x) d x+\lambda_{0} \int_{0}^{1} h(x) d x=0
\end{array}\right.
$$

3rd step, maxima are characteristic functions: Let us fix $\varepsilon \in(0,1 / 2)$. We are going to prove that the set $A_{\varepsilon}=\left\{x \in \Omega \mid \varepsilon \leq a^{*}(x) \leq 1-\varepsilon\right\}$ has zero measure for every $\varepsilon>0$ which obviously implies that $a^{*}$ is a characteristic function. Let us assume, for a contradiction, $\left|A_{\varepsilon}\right|>0$ and let us use the optimality conditions (3.5). We choose $h$ with a support in $A_{\varepsilon}$ : it is clearly admissible (we refer e.g. to [7], [4] for the complete description of the cone of admissible functions for the set $\mathcal{A}_{l}$ ). This implies:

$$
\sum_{k \in I\left(a^{*}\right)} \lambda_{k} \phi_{k}^{2}(x)+\lambda_{0}=0, \text { for almost every } x \in A_{\varepsilon}
$$


If $A_{\varepsilon}$ has positive measure, this equality can be extended to the whole interval by analyticity of the eigenfunctions. But such an identity is impossible since the system of functions

$$
\left\{1, \phi_{1}^{2}, \ldots, \phi_{k}^{2}\right\}=\left\{1,2 \sin ^{2} n_{1} \pi x, \ldots, 2 \sin ^{2} n_{k} \pi x\right\} \text { is linearly independent. }
$$

Consequently, for all $\varepsilon, A_{\varepsilon}$ has zero measure which proves the desired result.

4th step, uniqueness and symmetry: $J_{N}$ is clearly a concave function as a minimum of linear functions. Therefore, if it would exist two distinct maxima $a_{1}^{*}$ and $a_{2}^{*}$, all the points in the segment $\left[a_{1}^{*}, a_{2}^{*}\right]$ would also be maxima. But, it is impossible, since we have proved in step 3 that all the maxima were extreme points of the convex $\mathcal{A}_{l}$. Now, uniqueness implies symmetry of the minimizer with respect to $1 / 2$ since $J_{N}(a(x))=J_{N}(a(1-x))$.

5th step, at most $N$ connected components: We recall that the maximum $a^{*}=\chi_{\omega^{*}}$ satisfies the optimality condition (3.5). Let us introduce:

$$
\Psi_{\Lambda}(x)=2 \sum_{k \in I\left(a^{*}\right)} \lambda_{k} \sin ^{2}(k \pi x) .
$$

The Lagrangian of the maximization problem can be written:

$$
L\left(a, \lambda_{0}, \Lambda\right)=\int_{0}^{1} a(x) \Psi_{\Lambda}(x) d x+\lambda_{0}\left(\int_{0}^{1} a(x) d x-l\right) .
$$

Now, for every admissible function $h$ and every $\varepsilon>0$, small enough, we have:

$$
L\left(\chi_{\omega^{*}}+\varepsilon h, \lambda_{0}, \Lambda\right)-L\left(\chi_{\omega^{*}}, \lambda_{0}, \Lambda\right) \leq 0
$$

which can be rewritten, thanks to the linearity of $L$ with respect to its first variable:

$$
L\left(h, \lambda_{0}, \Lambda\right) \leq 0, \text { for } h \text { admissible }
$$

Now, we can choose as admissible $h$ a function satisfying:

$$
\begin{aligned}
& \forall x \in \omega^{*}, h(x) \leq 0 \\
& \forall x \in \omega^{* c}, h(x) \geq 0 \\
& \int_{0}^{1} h(x) d x=0
\end{aligned}
$$

For such a choice, we get:

$$
\begin{aligned}
& \forall x \in \omega^{*}, \Psi_{\Lambda}+\lambda_{0} \geq 0 \\
& \forall x \in \omega^{* c}, \Psi_{\Lambda}+\lambda_{0} \leq 0
\end{aligned}
$$

By continuity of $\psi_{\Lambda}$, equations (3.8) imply that for all $x \in \partial \omega^{*} \cap(0,1)$,

$$
\psi_{\Lambda}(x)+\lambda_{0}=0 .
$$

Now:

$$
\psi_{\Lambda}(x)=\sum_{k \in I\left(a^{*}\right)} \lambda_{k}(1-\cos (2 k \pi x))=1-\sum_{k \in I\left(a^{*}\right)} \lambda_{k} T_{k}(\cos 2 \pi x)
$$

where $T_{k}$ is the $k$-th Tchebyshev polynomial. Therefore, $\psi_{\Lambda}(x)$ is a polynomial in $\cos 2 \pi x$ of degree less or equal to $N$ and the equation $\psi_{\Lambda}(x)+\lambda_{0}=0$ has at most 
$2 N$ solutions in ]0,1[. Consequently, $\omega^{*}$ has at most $N$ connected components unless if $\omega^{*}$ contains an interval of the kind $[0, \eta]$ (and also $[1-\eta, 1]$ by symmetry). But this last case cannot happen since, for every small $\beta>0$ and for every integer $n$, $\int_{0}^{\eta} \phi_{n}(x) d x<\int_{\beta}^{\beta+\eta} \phi_{n}(x) d x$.

The equation (3.9) show that the optimal domain $\omega^{*}$ is a level set of the function $\Psi_{\Lambda}$ but, of course, it remains to find the Lagrange multipliers $\Lambda=\left(\lambda_{k}\right)_{k \in I\left(a^{*}\right)}$ and also to find $I\left(a^{*}\right)$. The following Theorem gives the answer to the second question and also gives a practical way to determine $\omega^{*}$ at least for small $l$.

THEOREM 3.2. For each integer $N$, there exists a real $l_{N} \leq 1$ such that for $l \leq l_{N}$, the optimal domain $\omega_{N}^{*}$ satisfies

$$
j_{1}\left(\omega_{N}^{*}\right)=j_{2}\left(\omega_{N}^{*}\right)=\ldots=j_{N}\left(\omega_{N}^{*}\right) .
$$

REMARK 1. The relations (3.10) together with the description of $\omega_{N}^{*}$ as a symmetric union of at most $N$ intervals yields a practical way to determine the optimum. Indeed, let us assume for example that $N=2 M$ is even. We write

$$
\omega_{N}^{*}=\bigcup_{k=1}^{M}\left[a_{k}-l_{k} / 2, a_{k}+l_{k} / 2\right] \cup \bigcup_{k=1}^{M}\left[1-a_{k}-l_{k} / 2,1-a_{k}+l_{k} / 2\right]
$$

then the relations (3.10) with the supplementary equality $\sum_{k=1}^{M} l_{k}=l / 2$ yields a $2 M \times 2 M$ non linear system whose (unique) solution gives the desired domain. We will use this remark later in section 4 .

REMARK 2. The relations (3.10) do not hold for any value of the constraint $l$ as it is shown by the following (numerical) example. Take $N=3$ and $l=0.9$ then the optimal domain is

$$
\omega_{3}^{*}=[0.0475707,0.3417644] \cup[0.3441937,0.6558063] \cup[0.6582356,0.9524293]
$$

which satisfies $j_{1}\left(\omega_{3}^{*}\right)>j_{2}\left(\omega_{3}^{*}\right)=j_{3}\left(\omega_{3}^{*}\right)$ and $J_{3}\left(\omega_{3}^{*}\right)=0.987672$ while for the best domain satisfying $j_{1}=j_{2}=j_{3}$, we have only $J_{3}(\omega)=0.987177$. Actually, we can see numerically that the constant $l_{N}$ decreases when $N$ increases.

Proof of Theorem 3.2 : The first idea consists in transposing the problem in finite dimension thanks to the following trick. Let $\mathcal{K}_{N}^{l}$ be the subset of $\mathbb{R}^{N}$ defined by

$$
\mathcal{K}_{N}^{l}=\left\{X=\left(x_{1}, x_{2}, \ldots, x_{N}\right), \text { s.t. } \exists a \in \mathcal{A}_{l} \text { with } x_{i}=j_{i}(a), i=1,2, \ldots, N\right\}
$$

We will also write $\mathcal{K}_{N}^{l}=\mathcal{K}$ when $N$ and $l$ are fixed, since no misunderstanding is possible. The set $\mathcal{K}$ is obviously convex and compact, since it is the image of $\mathcal{A}_{l}$ by the linear (continuous) functional $a \mapsto\left(j_{1}(a), j_{2}(a), \ldots, j_{N}(a)\right)$. We will give, in section 5 some supplementary properties of $\mathcal{K}$.

The first bissectrix $\Delta=\left\{X \in \mathbb{R}^{N}, x_{1}=x_{2}=\ldots=x_{N}\right\}$ meets $\mathcal{K}$ (take $a=l$ a constant: we have $j_{i}(a)=l$ for all $\left.i\right)$. Therefore, we can introduce the point $X^{*}$, the furthest point of $\Delta \cap \mathcal{K}$ :

$$
X^{*}=\left(x^{*}, x^{*}, \ldots, x^{*}\right) \text { with } x^{*}=\max \{x \operatorname{such} \text { that } X=(x, x, \ldots, x) \in \Delta \cap \mathcal{K}\} .
$$

Note that $x^{*}>l$ since $a=l$ cannot be the maximizer of $J_{N}$. The claim in Theorem 3.2 is equivalent to say that $X^{*}$ solves the maximization problem:

$$
G\left(X^{*}\right)=\max _{X \in \mathcal{K}} G(X) \quad \text { where } G(X)=\min _{1 \leq k \leq N}\left\{x_{k}\right\} .
$$


A geometrical interpretation of (3.12) consists in saying that there is no point of $\mathcal{K}$ in the quadrant

$$
\mathcal{Q}=\left\{X=\left(x_{1}, x_{2}, \ldots, x_{N}\right) \in \mathbb{R}^{N} ; x_{i}>x^{*}, i=1,2, \ldots, N\right\} .
$$

To prove Theorem 3.2, we argue by contradiction. We will prove that if $\mathcal{Q} \cap \mathcal{K}$ is not empty, there exists a point of the bissectrix $\Delta$ in $\mathcal{Q} \cap \mathcal{K}$ (at least for $l$ small enough) which contradicts the fact that $X^{*}$ maximizes $G$ on $\Delta \cap \mathcal{K}$. For that purpose, we introduce $X_{k}$ to be the furthest point of $\mathcal{K}$ in the direction of $x_{k}$ (the $k$-th coordinate). Actually, we can determine $X_{k}$. Indeed, $X_{k}$ is obtained by solving the maximization problem: find $a \in \mathcal{A}_{l}$ which maximizes $\int_{0}^{1} a(x) \sin ^{2} k \pi x d x$. It follows from the proof of Theorem 3.1 (steps 2,3,5 and, in particular relation (3.9)) that the maximizer is a characteristic function $\chi_{\omega_{k}^{*}}$, level set of the function $\sin ^{2} k \pi x$. Therefore,

$$
\omega_{k}^{*}=\bigcup_{j=0}^{k-1}\left[\frac{2 j+1}{2 k}-\frac{l}{2 k}, \frac{2 j+1}{2 k}+\frac{l}{2 k}\right] .
$$

We can easily deduce the coordinates of $X_{k}$. For $k=1$,

$$
j_{m}\left(\omega_{1}^{*}\right)=l+(-1)^{m+1} \frac{\sin m \pi l}{m \pi}
$$

while, for $k \geq 2$ :

$$
j_{m}\left(\omega_{k}^{*}\right)=l-\sum_{j=0}^{k-1} \frac{1}{m \pi} \sin \frac{m \pi l}{k} \cos \frac{2 m \pi(2 j+1)}{2 k} .
$$

Using $\sum_{j=0}^{k-1} \cos \frac{m \pi(2 j+1)}{k}=0$ for $m \neq k$, this yields

$$
j_{m}\left(\omega_{k}^{*}\right)=l \text { if } m \neq k \text { and } j_{k}\left(\omega_{k}^{*}\right)=l+\frac{1}{\pi} \sin \pi l .
$$

For simplicity, let us put the origin at $X^{*}$. In this case, to sum up, the vertices of the set $\mathcal{K}$ in the direction of the coordinate axis are the points:

$$
X_{1}=\left(\begin{array}{c}
h \\
\beta_{2} \\
\beta_{3} \\
\vdots \\
\beta_{N}
\end{array}\right) X_{2}=\left(\begin{array}{c}
-\alpha \\
h \\
-\alpha \\
\vdots \\
-\alpha
\end{array}\right) \quad X_{3}=\left(\begin{array}{c}
-\alpha \\
-\alpha \\
h \\
-\alpha \\
\vdots
\end{array}\right) \ldots X_{N}=\left(\begin{array}{c}
-\alpha \\
-\alpha \\
\vdots \\
-\alpha \\
h
\end{array}\right)
$$

where

$$
\begin{aligned}
& \alpha=x^{*}-l>0, \\
& h=l+\frac{\sin \pi l}{\pi}-x^{*}>0, \\
& \text { and } \beta_{i}=l+(-1)^{i+1} \frac{\sin i \pi l}{i \pi}-x^{*}, i=2, \ldots, N .
\end{aligned}
$$

As explained above, let us assume (for a contradiction) that there is a point $X_{0}=$ $\left(x_{1}^{0}, \ldots, x_{N}^{0}\right)^{T}$ in $\mathcal{Q} \cap \mathcal{K}$. We are looking for a point $X$, convex combination of $X^{*}=O$, $X_{0}, X_{1}, X_{2}, \ldots, X_{N}$ (which ensures that $X$ will be in $\mathcal{K}$ ) that we want to be in the 
set $\mathcal{Q}$ and on the bissectrix $\Delta$. Existence of such a point would lead to a contradiction since $X^{*}$ maximizes $G$ on $\Delta \cap \mathcal{K}$. We write $X$ as

$$
X=(1-s) O+t X_{0}+\sum_{i=1}^{N} \lambda_{i} X_{i}
$$

where

$$
0<s \leq 1,0 \leq t \leq 1,0 \leq \lambda_{i} \leq 1, t+\sum_{i=1}^{N} \lambda_{i}=s
$$

Let us first express that $X$ must belong to $\Delta$. Writing $x_{1}=x_{2}, x_{1}=x_{3}$, etc... this yields the following system:

$$
\left\{\begin{array}{c}
t x_{1}^{0}+\lambda_{1} h-\lambda_{2} \alpha=t x_{2}^{0}+\lambda_{1} \beta_{2}+\lambda_{2} h \\
t x_{1}^{0}+\lambda_{1} h-\lambda_{3} \alpha=t x_{3}^{0}+\lambda_{1} \beta_{3}+\lambda_{3} h \\
\vdots \\
t x_{1}^{0}+\lambda_{1} h-\lambda_{N} \alpha=t x_{N}^{0}+\lambda_{1} \beta_{N}+\lambda_{N} h .
\end{array}\right.
$$

Summing these relations and using (3.16) gives:

$$
(N-1) t x_{1}^{0}+(N-1) \lambda_{1} h-\alpha\left(s-t-\lambda_{1}\right)=t \sum_{i=2}^{N} x_{i}^{0}+\lambda_{1} \sum_{i=2}^{N} \beta_{i}+h\left(s-t-\lambda_{1}\right)
$$

what can be written, thanks to (3.15):

$$
t\left[\left(N x_{1}^{0}-\sum_{i=1}^{N} x_{i}^{0}\right)+\frac{\sin \pi l}{\pi}\right]+\lambda_{1}\left[\sum_{i=2}^{N}\left(h-\beta_{i}\right)+\frac{\sin \pi l}{\pi}\right]=\frac{\sin \pi l}{\pi} s .
$$

Let us introduce

$$
\Sigma=\sum_{i=2}^{N}\left(h-\beta_{i}\right)+\frac{\sin \pi l}{\pi}=N \frac{\sin \pi l}{\pi}+\sum_{i=2}^{N}(-1)^{i} \frac{\sin i \pi l}{i \pi} .
$$

From now, we will assume $l \leq 1 /(2 N)$. Then, $\Sigma$ is clearly positive. We get $\lambda_{1}$ from (3.18):

$$
\lambda_{1}=\left(\frac{\sin \pi l}{\pi} s-t\left[\left(N x_{1}^{0}-\sum_{i=1}^{N} x_{i}^{0}\right)+\frac{\sin \pi l}{\pi}\right]\right) / \Sigma .
$$

Replacing in each equation (3.17) gives the value of $\lambda_{k}$ :

$$
\lambda_{k}=\frac{t\left(x_{1}^{0}-x_{k}^{0}\right)}{\frac{\sin \pi l}{\pi}}+\frac{\lambda_{1}\left(h-\beta_{k}\right)}{\frac{\sin \pi l}{\pi}} .
$$

We now use $h-\beta_{k}=\frac{\sin \pi l}{\pi}+(-1)^{k} \frac{\sin k \pi l}{k \pi}$ (see (3.15)) and we introduce normalized coordinates: $y_{k}^{0}:=\frac{x_{k}^{0}}{\frac{\sin \pi l}{\pi}}$. Therefore, (3.19) and (3.20) can be rewritten

$$
\begin{aligned}
& \lambda_{1}=\frac{\frac{\sin \pi l}{\pi}}{\Sigma}\left(s-t-t\left(N y_{1}^{0}-\sum_{i=1}^{N} y_{i}^{0}\right)\right) \\
& \lambda_{k}=t\left(y_{1}^{0}-y_{k}^{0}\right)+\frac{\frac{\sin \pi l}{\pi}+(-1)^{k} \frac{\sin k \pi l}{k \pi}}{\Sigma}\left(s-t-t\left(N y_{1}^{0}-\sum_{i=1}^{N} y_{i}^{0}\right)\right) .
\end{aligned}
$$


We also compute $x_{1}\left(=x_{2}=\ldots=x_{N}\right)$, we easily obtain

$$
x_{1}=s\left(\frac{\left(\frac{\sin \pi l}{\pi}\right)^{2}}{\Sigma}-\alpha\right)+t\left(x_{1}^{0}+\alpha-\frac{\left(\frac{\sin \pi l}{\pi}\right)^{2}}{\Sigma}-\frac{\frac{\sin \pi l}{\pi}}{\Sigma}\left(N x_{1}^{0}-\sum_{i=1}^{N} x_{i}^{0}\right)\right) .
$$

We recall that we want $\lambda_{1} \geq 0$. So, we have the first necessary condition:

$$
Q_{1}:=s-t\left(1+N y_{1}^{0}-\sum_{i=1}^{N} y_{i}^{0}\right) \geq 0 .
$$

We will now express that every $\lambda_{k}$ has also to be non-negative. For that purpose, we introduce the (non positive) number:

$$
-\xi:=\min _{1 \leq k \leq N}\left(y_{1}^{0}-y_{k}^{0}\right)
$$

and we assume the second necessary condition:

$$
\xi t \leq \frac{\frac{\sin \pi l}{\pi}-\frac{\sin 3 \pi l}{3 \pi}}{\Sigma} Q_{1} .
$$

(3.24) together with (3.25) imply

$$
\forall k, t\left(y_{1}^{0}-y_{k}^{0}\right) \geq-\xi t \geq \frac{\frac{\sin 3 \pi l}{3 \pi}-\frac{\sin \pi l}{\pi}}{\Sigma} Q_{1} .
$$

Therefore, from (3.20), it comes

$$
\lambda_{k} \geq \frac{\frac{\sin 3 \pi l}{3 \pi}+(-1)^{k} \frac{\sin k \pi l}{k \pi}}{\Sigma} Q_{1} .
$$

For $k=2,3,4$, it is clear that (3.26) with (3.23) imply $\lambda_{k} \geq 0$. For higher values of $k$, it is also true. Indeed, since $x \mapsto \sin x / x$ is decreasing for $x<\pi / 2$, we have $\sin (3 \pi l) /(3 \pi) \geq \sin (k \pi l) /(k \pi)$ for $3 \leq k \leq N$.

In conclusion, if we assume (3.23), (3.25) and $l<1 /(2 N)$, then $\lambda_{k} \geq 0$, for all $k \leq N$. Let us set,

$$
\gamma=\frac{t}{s} \quad Y=1+N y_{1}^{0}-\sum_{i=1}^{N} y_{i}^{0} .
$$

Then (3.23) can be rewritten $1-\gamma Y \geq 0$ and is obviously true for $\gamma$ small enough. In the same way, (3.25) is

$$
\xi \gamma \leq \frac{\frac{\sin \pi l}{\pi}-\frac{\sin 3 \pi l}{3 \pi}}{\Sigma}(1-\gamma Y)
$$

what is also true for $\gamma$ small since $\frac{\sin \pi l}{\pi}-\frac{\sin 3 \pi l}{3 \pi}>0$.

It remains to check that $x_{1}$, given by (3.22), is positive. With the previous notations,

$$
x_{1}=\gamma\left(x_{1}^{0}-Y \frac{\left(\frac{\sin \pi l}{\pi}\right)^{2}}{\Sigma}+\alpha\right)+\frac{\left(\frac{\sin \pi l}{\pi}\right)^{2}}{\Sigma}-\alpha
$$


therefore, if we are able to prove that $\frac{\left(\frac{\sin \pi l}{\pi}\right)^{2}}{\Sigma}-\alpha>0$, it will imply that $x_{1}>0$ for $\gamma$ small enough.

Now, when $l \rightarrow 0$

$$
\frac{\left(\frac{\sin \pi l}{\pi}\right)^{2}}{\Sigma} \sim\left\{\begin{array}{cl}
\frac{l}{N} & \text { if } N \text { is odd } \\
\frac{l}{N+1} & \text { if } N \text { is even }
\end{array}\right.
$$

On the other hand, the proof of Theorem 4.1 below shows that, when $l \rightarrow 0$,

$$
x^{*} \sim j_{1}\left(\bigcup_{k=1}^{N}\left[\frac{k}{N+1}-\frac{l}{2 N}, \frac{k}{N+1}+\frac{l}{2 N}\right]\right)=l-\frac{1}{\pi} \sin \frac{\pi l}{2 N} \sum_{k=1}^{N} \cos \frac{2 k \pi}{N+1} .
$$

Since $\sum_{k=1}^{N} \cos \frac{2 k \pi}{N+1}=-1$, this yields

$$
\alpha=x^{*}-l \sim \frac{1}{\pi} \sin \frac{\pi l}{2 N} \sim \frac{l}{2 N}
$$

and the result follows from the comparison with (3.27). This finishes the proof of Theorem 3.2.

4. The spillover phenomenon. We are going to describe more precisely the optimal domain $\omega_{N}^{*}$ when the length constraint $l$ goes to zero. According to Theorem $3.1, \omega_{N}^{*}$ is symmetric and has, at most, $N$ connected components. Therefore,

in the case $N$ even we write $N=2 K$ and there exists $0<\alpha_{1}<\alpha_{2}<\cdots<\alpha_{K}<$ $1 / 2$ and $l_{1}, l_{2}, \ldots, l_{K} \geq 0$ such that :

$$
\omega_{N}^{*}=\left(\bigcup_{i=1}^{K}\left[\alpha_{i}-l_{i} / 2, \alpha_{i}+l_{i} / 2\right]\right) \bigcup\left(\bigcup_{i=1}^{K}\left[1-\alpha_{i}-l_{i} / 2,1-\alpha_{i}+l_{i} / 2\right]\right)
$$

in the case $N$ odd we write $N=2 K+1$ and there exists $0<\alpha_{1}<\alpha_{2}<\cdots<$ $\alpha_{K}<1 / 2$ and $l_{1}, l_{2}, \ldots, l_{K+1} \geq 0$ such that :

$$
\begin{gathered}
\omega_{N}^{*}=\left(\bigcup_{i=1}^{K}\left[\alpha_{i}-l_{i} / 2, \alpha_{i}+l_{i} / 2\right]\right) \bigcup\left[1 / 2-l_{K+1} / 2,1 / 2+l_{K+1} / 2\right] \ldots \\
\ldots \bigcup\left(\bigcup_{i=1}^{K}\left[1-\alpha_{i}-l_{i} / 2,1-\alpha_{i}+l_{i} / 2\right]\right)
\end{gathered}
$$

The main result of this section is the following:

THEOREM 4.1. When $l$ goes to 0 , the optimal domain for the $N$ first modes $\omega_{N}^{*}$ concentrates around the nodes of the $N+1$-th eigenfunction.

More precisely, when $l \rightarrow 0^{+}$:

$$
\forall i, \quad \alpha_{i}(l) \rightarrow \frac{i}{N+1}, \quad l_{i}(l) \sim \frac{l}{N}
$$

For sake of simplicity, we are going to prove this result in the case $N=2 K$ even, the case $N$ odd is exactly similar. We now express that $\omega_{N}^{*}$ satisfies

$$
j_{1}\left(\omega_{N}^{*}\right)=j_{2}\left(\omega_{N}^{*}\right)=\cdots=j_{N}\left(\omega_{N}^{*}\right) .
$$


This yields:

$$
\left\{\begin{array}{l}
\sum_{i=1}^{K}\left[\frac{1}{n \pi} \sin n \pi l_{i} \cos 2 n \pi \alpha_{i}-\frac{1}{(n-1) \pi} \sin (n-1) \pi l_{i} \cos (2 n-2) \pi \alpha_{i}\right]=0 \\
\quad \text { for } n=2,3, \ldots, N .
\end{array}\right.
$$

Asymptotically, when $l$ goes to 0 , the $\left(\alpha_{i}\right)$ 's and the $\left(l_{i}\right)^{\prime}$ 's are therefore solution of the linearized system (in a neighborhood of $l=0$ ) :

$$
\sum_{i=1}^{K} l_{i}\left(\cos 2 n \pi \alpha_{i}-\cos (2 n-2) \pi \alpha_{i}\right)=0, \quad \text { for } n=2,3, \ldots, N
$$

Now if $\left(l_{p}\right)$ is a sequence which tends to 0 , after extracting a finite number of subsequence, we can write $l_{i}^{p}=t_{i}\left(l_{p}\right) \cdot l_{p}$ with $t_{i}\left(l_{p}\right) \in[0,1]$ converging to $t_{i}$ and $a_{i}\left(l_{p}\right)$ which converge to $a_{i}$. The system (4.3) leads to the following system

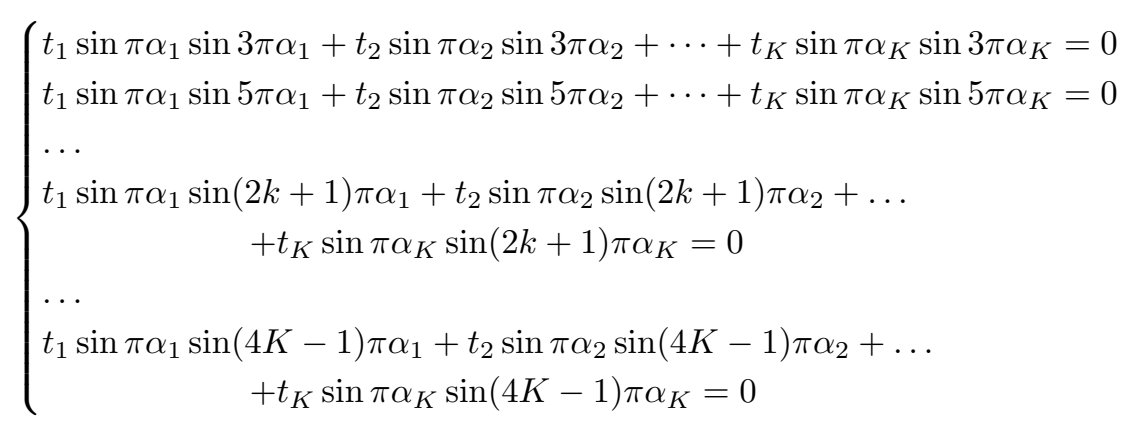

where we have to add the supplementary equation:

$$
t_{1}+t_{2}+\ldots t_{K}=1 / 2 .
$$

This new system can be viewed as a $(2 K-1) \times(2 K-1)$ linear system with unknowns $t_{1}, t_{2}, \ldots, t_{K}$. Of course these unknowns cannot be all equal to zero because of the supplementary equation (4.5). Therefore the matrix $A$ of system (4.4) must be of rank less or equal to $K-1$. It means that:

all determinants $K \times K$ extracted from $A$ are equal to 0 .

Let us denote by $\mathcal{L}_{1}, \mathcal{L}_{2}, \ldots, \mathcal{L}_{2 K-1}$, the lines of the matrix $A$ :

$$
A=\left(\begin{array}{ccc}
\sin \pi \alpha_{1} \sin 3 \pi \alpha_{1} & \ldots & \sin \pi \alpha_{K} \sin 3 \pi \alpha_{K} \\
\sin \pi \alpha_{1} \sin 5 \pi \alpha_{1} & \ldots & \sin \pi \alpha_{K} \sin 5 \pi \alpha_{K} \\
\vdots & & \vdots \\
\sin \pi \alpha_{1} \sin (4 K-1) \pi \alpha_{1} & \ldots & \sin \pi \alpha_{K} \sin (4 K-1) \pi \alpha_{K}
\end{array}\right) \begin{aligned}
& \rightarrow \mathcal{L}_{1} \\
& \rightarrow \mathcal{L}_{2} \\
& \vdots \\
& \rightarrow \mathcal{L}_{2 K-1}
\end{aligned}
$$

Now, let us compute the following $K \times K$ determinant :

$$
D_{0}=\operatorname{det}\left(\mathcal{L}_{K}+\mathcal{L}_{K}, \mathcal{L}_{K-1}+\mathcal{L}_{K+1}, \ldots, \mathcal{L}_{1}+\mathcal{L}_{2 K-1}\right) .
$$

According to (4.6), $D_{0}=0$.

On the other hand, for $k=0,1, \ldots, K-1$ and $i=1,2, \ldots, K$ :

$$
\sin (2(K-k)+1) \pi \alpha_{i}+\sin (2(K+k)+1) \pi \alpha_{i}=2 \sin (2 K+1) \pi \alpha_{i} \cos 2 k \pi \alpha_{i} .
$$


Therefore :

$$
D_{0}=2^{K}\left(\prod_{i=1}^{K} \sin \pi \alpha_{i}\right)\left(\prod_{i=1}^{K} \sin (2 K+1) \pi \alpha_{i}\right) D_{0}^{\prime}
$$

where $D_{0}^{\prime}$ is the determinant :

$$
D_{0}^{\prime}=\left|\begin{array}{cccc}
1 & 1 & \ldots & 1 \\
\cos 2 \pi \alpha_{1} & \cos 2 \pi \alpha_{2} & \ldots & \cos 2 \pi \alpha_{K} \\
\vdots & \vdots & & \vdots \\
\cos 2(K-1) \pi \alpha_{1} & \cos 2(K-1) \pi \alpha_{2} & \ldots & \cos 2(K-1) \pi \alpha_{K}
\end{array}\right|
$$

This determinant $D_{0}^{\prime}$ can be computed thanks to Tchebyshev polynomials $T_{k}$, already introduced $\left(\cos k x=T_{k}(\cos x)\right)$ with $T_{k}$ of degree $k$ and highest degree term is $2^{k-1} X^{k}, k \geq 1$. Since the family $\left(T_{k}\right)_{0 \leq k \leq K-1}$ is a basis of polynomial of degree less than $K-1$, we can write :

$$
D_{0}^{\prime}=\left(\prod_{k=1}^{K-1} 2^{k-1}\right)\left|\begin{array}{cccc}
1 & 1 & \ldots & 1 \\
\cos 2 \pi \alpha_{1} & \cos 2 \pi \alpha_{2} & \ldots & \cos 2 \pi \alpha_{K} \\
\vdots & \vdots & & \vdots \\
\cos ^{K-1} 2 \pi \alpha_{1} & \cos ^{K-1} 2 \pi \alpha_{2} & \ldots & \cos ^{K-1} 2 \pi \alpha_{K}
\end{array}\right|
$$

Now this last determinant is the so-called Van der Mond determinant, so :

$$
\begin{aligned}
D_{0}^{\prime} & =2^{\frac{(K-1)(K-2)}{2}} \prod_{1 \leq i<j \leq K}\left(\cos 2 \pi \alpha_{i}-\cos 2 \pi \alpha_{j}\right) \\
& =2^{\frac{(K-1)(K-2)}{2}} \prod_{1 \leq i<j \leq K} 2\left(\cos ^{2} \pi \alpha_{i}-\cos ^{2} \pi \alpha_{j}\right) \\
& =2^{(K-1)^{2}} \prod_{1 \leq i<j \leq K}\left(\cos ^{2} \pi \alpha_{i}-\cos ^{2} \pi \alpha_{j}\right)
\end{aligned}
$$

This last equality shows that $D_{0}^{\prime}$ cannot vanish (we recall that the $\left(\alpha_{i}\right)$ is an increasing sequence with $\left.0<\pi \alpha_{i}<\pi / 2\right)$. Moreover, the product $\prod_{i=1}^{K} \sin \pi \alpha_{i}$ is not zero since, for all $\left.i, \alpha_{i} \in\right] 0,1 / 2[$.

In conclusion, there exists $p \in\{1,2, \ldots, K\}$ such that $\sin (2 K+1) \pi \alpha_{p}=0$, it means that there exists $q \in\{1,2, \ldots, K\}$ such that $\alpha_{p}=\frac{q}{2 K+1}$.

Let us now compute, in the same way, the following $K \times K$ determinant :

$$
D_{1}=\operatorname{det}\left(\mathcal{L}_{K}+\mathcal{L}_{K}, \mathcal{L}_{K-1}+\mathcal{L}_{K+1}, \ldots, \mathcal{L}_{2}+\mathcal{L}_{2 K-2}, \mathcal{L}_{1}\right) .
$$

For the same reason as above $D_{1}=0$. Grouping the sin terms yields:

$$
D_{1}=2^{K}\left(\prod_{i=1}^{K} \sin \pi \alpha_{i}\right)\left(\prod_{i=1,2, \ldots, K ; i \neq p} \sin (2 K+1) \pi \alpha_{i}\right) D_{1}^{\prime}
$$

where $D_{1}^{\prime}$ is the determinant :

$$
D_{1}^{\prime}=\left|\begin{array}{ccccc}
1 & \ldots & 0 & \ldots & 1 \\
\cos 2 \pi \alpha_{1} & \ldots & 0 & \ldots & \cos 2 \pi \alpha_{K} \\
\vdots & & \vdots & & \vdots \\
\cos 2(K-1) \pi \alpha_{1} & \ldots & \sin \frac{3 \pi q}{2 K+1} & \ldots & \cos 2(K-1) \pi \alpha_{K}
\end{array}\right|
$$


Developing $D_{1}^{\prime}$ with respect to its $p^{\text {th }}$ column, it appears a $K-1 \times K-1$ determinant similar to $D_{0}^{\prime}$. This shows that $D_{1}^{\prime}$ does not vanish. Therefore, there exists $r \neq p$ such that $\sin (2 K+1) \pi \alpha_{r}=0$, what means that there exists $s \in\{1,2, \ldots, K\}$ such that $\alpha_{r}=\frac{s}{2 K+1}$.

By computing successively, using the same method as for $D_{1}$, the determinants $D_{2}, D_{3}, \ldots, D_{K-1}$, with :

$$
\begin{aligned}
& D_{2}=\operatorname{det}\left(\mathcal{L}_{K}+\mathcal{L}_{K}, \mathcal{L}_{K-1}+\mathcal{L}_{K+1}, \ldots, \mathcal{L}_{3}+\mathcal{L}_{2 K-3}, \mathcal{L}_{2}, \mathcal{L}_{1}+\mathcal{L}_{2 K-1}\right) \\
& D_{3}=\operatorname{det}\left(\mathcal{L}_{K}+\mathcal{L}_{K}, \mathcal{L}_{K-1}+\mathcal{L}_{K+1}, \ldots, \mathcal{L}_{3}, \mathcal{L}_{2}+\mathcal{L}_{2 K-2}, \mathcal{L}_{1}+\mathcal{L}_{2 K-1}\right) \\
& \quad \vdots \\
& D_{K-1}=\operatorname{det}\left(\mathcal{L}_{K}+\mathcal{L}_{K}, \mathcal{L}_{K-1}, \mathcal{L}_{K-2}+\mathcal{L}_{K+2} \ldots, \mathcal{L}_{2}+\mathcal{L}_{2 K-2}, \mathcal{L}_{1}+\mathcal{L}_{2 K-1}\right)
\end{aligned}
$$

we can show that, for all $1 \leq i \leq K$, there exists $p_{i}$ such that $\alpha_{i}=\frac{p_{i} \pi}{2 K+1}$. Since the sequence $\left(\alpha_{i}\right)$ is increasing, we have :

$$
\forall i \in\{1,2, \ldots, K\} \quad \alpha_{i}=\frac{i \pi}{2 K+1}
$$

Let us now show that the rank of the matrix $A$ is exactly $K-1$. For that purpose, we compute the determinant $D$ obtained from $A$ by taking the $K-1$ first lines and removing the last column :

$$
D=\left[\prod_{i=1}^{K-1} \sin \frac{i \pi}{2 K+1}\right] \operatorname{det}\left(\left(\sin \frac{(2 i+1) \pi j}{2 K+1}\right)_{1 \leq i, j \leq K-1}\right)
$$

Let us denote by $U_{n}$ the Tchebyshev's polynomial of second kind $: \sin n \theta=\sin \theta U_{n}(\cos \theta)$. We obtain :

$$
D=\left[\prod_{i=1}^{K-1} \sin \frac{i \pi}{2 K+1}\right] \operatorname{det}\left(\left(U_{j}\left(\sin \frac{(2 i+1) \pi}{2 K+1}\right)\right)_{1 \leq i, j \leq K-1}\right) .
$$

Since $U_{n}$ has for first term $2^{n} X^{n}, n \geq 1$, we get :

$$
D=2^{\frac{(K-2)(K-3)}{2}}\left(\prod_{i=1}^{K-1} \sin \frac{i \pi}{2 K+1}\right)\left(\prod_{1 \leq i<j \leq K-1}\left(\cos \frac{(2 i+1) \pi}{2 K+1}-\cos \frac{(2 j+1) \pi}{2 K+1}\right)\right)
$$

This proves that $D \neq 0$ and $A$ has rank $K-1$. Therefore, its kernel is of dimension one.

Let us now show that $(1,1, \ldots, 1)^{t}$ belongs to the kernel of $A$. For that purpose, we compute, for $1 \leq k \leq 2 K-1$ the sum :

$$
S_{k}=2 \sum_{l=1}^{K} \sin \frac{l \pi}{2 K+1} \sin \frac{(2 k+1) l \pi}{2 K+1}=\sum_{l=1}^{K}\left[\cos \frac{2 l k \pi}{2 K+1}-\cos \frac{2 l(k+1) \pi}{2 K+1}\right] .
$$

Using, $\sum_{l=1}^{K} \cos l \theta=\cos \frac{(K+1) \theta}{2} \cdot \frac{\sin \frac{K \theta}{2}}{\sin \frac{\theta}{2}}$ it comes that $S_{k}=0$ if and only if :

$$
\cos \frac{(K+1) k \pi}{2 K+1} \sin \frac{K k \pi}{2 K+1} \sin \frac{(k+1) \pi}{2 K+1}=\ldots
$$




$$
\ldots=\cos \frac{(K+1)(k+1) \pi}{2 K+1} \sin \frac{K(k+1) \pi}{2 K+1} \sin \frac{k \pi}{2 K+1 .}
$$

This last equality is easy to check.

In conclusion, since $\left(t_{1}, t_{2}, \ldots, t_{K}\right)^{t}$ belongs to the kernel of $A$ and $t_{1}+t_{2}+\cdots+$ $t_{K}=1 / 2$, we get $t_{1}=t_{2}=\cdots=t_{K}=1 /(2 K)$. Finally, for every subsequence $\left(l_{k}\right)$ converging to $0,\left(t_{i}\left(l_{k}\right)\right)$ has a unique accumulation point, the whole sequence $t_{i}(l)$ converges to that point $1 / K$. In the same way, the functions $\alpha_{i}(l)$ converge to $i /(K+1)$ when $l$ goes to 0 .

\section{Comments.}

5.1. Possible remedies. To avoid the spillover phenomenon which is described here, we can imagine different possible strategies. The first one is obviously to take into account all eigenmodes, possibly with different weigths for each (e.g. weigths decreasing with the rank of the mode).

Another possibility could be inspired by the introduction of an artificial (numerical) viscosity like in papers [23], [24]. For the 1-D wave equation, these authors choose to introduce a semi-discrete term coming from $-h^{2} u_{x x t}$. This has the great advantage to keep the decay properties of the discrete equation which are generally lost under the semi-discrete finite-differences scheme. It would be very interesting to see what is the impact of this viscosity term in the context of our paper.

5.2. Larger values of $l$. It is essentially for technical reasons that the "spillover" phenomenon is described in the case $l \rightarrow 0$. Actually, this phenomenon holds for most values of $l$. The following Figure 5.1 shows numerical results for $N=3$. The
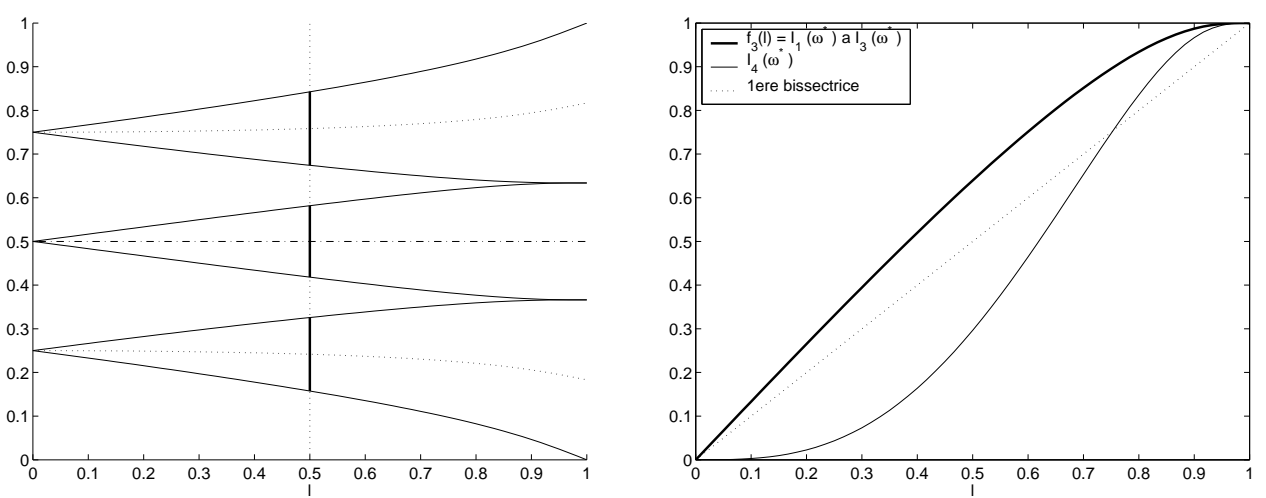
$j_{4}\left(\omega_{3}^{*}\right)$

FIG. 5.1. left: the optimal domain $\omega_{3}^{*}$ (read vertically); right: graphs of $J_{3}\left(\omega_{3}^{*}\right)$ (bold face) and

left picture shows the optimal domain $\omega_{3}^{*}$ for each value of $l$. One obtains $\omega_{3}^{*}$ as the intersection of the vertical line $x=l$ with the interior of the three peeks. The right picture shows in boldface the graph of $l \mapsto J_{3}\left(\omega_{3}^{*}\right)$ and below, in medium, the graph of $l \mapsto j_{4}\left(\omega_{3}^{*}\right)$. We see that, in any case (unless for $l$ close to 1 ), $\omega_{3}^{*}$ has a very bad behaviour for the fourth eigenmode. One can find more examples in [14].

5.3. More about the set $\mathcal{K}$. For similar optimization problems, it is interesting to have a more precise description of the set $\mathcal{K}$ which is introduced in (3.11). In particular, a characterization of its boundary can be very useful. Let $X$ be a point in 
$\mathcal{K}$. Then $X$ belongs to $\partial \mathcal{K}$ if and only if there exists a unit vector $\mathbf{n}$ such that:

$$
\forall Y \in \mathcal{K} \quad(Y-X, \mathbf{n}) \leq 0 .
$$

Writing $\mathbf{n}=\left(n_{1}, n_{2}, \ldots, n_{N}\right)^{T}, X=\left(j_{1}(a), \ldots, j_{N}(a)\right)^{T}$ and $Y=\left(j_{1}(b), \ldots, j_{N}(b)\right)^{T}$, the latter reads

$$
\forall b \in \mathcal{A}_{l} \quad \sum_{k=1}^{N} n_{k} j_{k}(b) \leq \sum_{k=1}^{N} n_{k} j_{k}(a)
$$

In other terms, $a$ is a maximizer of the functional $b \mapsto \sum_{k=1}^{N} n_{k} j_{k}(b)$. According to the proof of Theorem 3.1, it follows that $a$ is necessarily the characteristic function of a set $\omega$ which is the union of at most $N+1$ intervals and which is symmetric with respect to $1 / 2$. The fact that we can have $N+1$ intervals here is due to the fact that intervals of the kind $[0, \eta]$ or $[1-\eta, 1]$ are allowed here (see step 5 in the proof of Theorem 3.1). Figure 5.2 (left) shows the set $\mathcal{K}_{2}$ for $l=0.3$. Its boundary
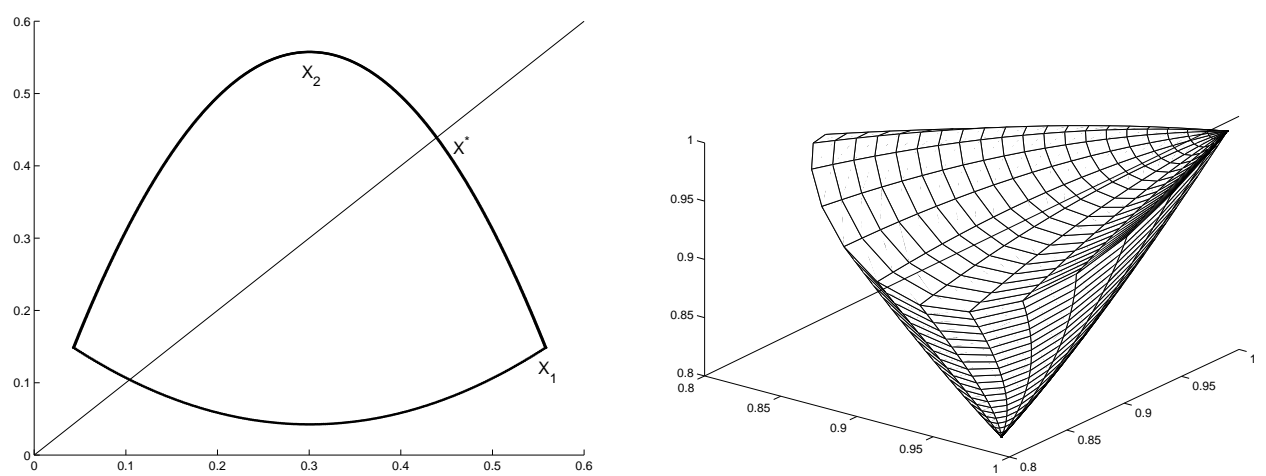

FIG. 5.2. left: the set $\mathcal{K}_{2}$ for $l=0.3$; right: the set $\mathcal{K}_{3}$ for $l=0.9$

is exactly the image of characteristic functions $\chi_{\omega}$ with $\omega$ is a symmetric (w.r.t. $1 / 2$ ) set obtained as an union of one, two or three intervals (in this last case two of the three intervals must touch 0 and 1). Figure 5.2 (right) shows the set $\mathcal{K}_{3}$ for $l=0.9$. Its boundary is obtained with a symmetric union of one, two, three or four intervals (in this last case two of the four intervals must touch 0 and 1 ). This picture shows a case where the first bissectrix does not cut the set $\mathcal{K}_{3}$ at the point which maximizes $\min \left(x_{1}, x_{2}, x_{3}\right)$, see Remark 2 .

5.4. Generalization to the two-dimensional case. The existence and uniqueness part of Theorem 3.1 can be easily generalized to more general domains $\Omega$ in higher dimension if the following property holds:

$$
\left\{\begin{array}{l}
\text { Let } \varphi_{1}, \varphi_{2}, \ldots, \varphi_{N} \text { be the (normalized) eigenfunctions of the Laplace } \\
\text { operator on } \Omega \text { with Dirichlet boundary conditions on } \partial \Omega \text {, then } \\
\varphi_{1}^{2}, \varphi_{2}^{2}, \ldots, \varphi_{N}^{2} \text { are linearly independent on } \omega \text {. }
\end{array}\right.
$$

Indeed, when we look at the proof of Theorem 3.1, we observe that it can easily be adapted to any dimension, the only technical point being (3.7).

The property (5.1) is obviously true for rectangles in two dimension or, more generally, parallelepiped in $N$-dimension, but the authors do not know if it holds for 
every domain (even for a disc). For a related result in one-dimension, see [19]. In this paper the authors prove that for a non homogeneous Sturm-Liouville eigenvalue problem, it happens very frequently that the $N$ first eigenfunctions, with $N \geq 3$, have linearly dependent squares on some non trivial interval. A transposition of this one-dimensional result to our case could lead to the following conjecture:

Open problem 1: Prove that for every domain $\Omega$, there exists a domain $\tilde{\Omega}$ close to $\Omega$ such that the square of a given number of eigenfunctions of the Laplace-Dirichlet operator on $\tilde{\Omega}$ are linearly dependent.

On the other hand, if the result (5.1) is wrong for some domain $\Omega$, one can also imagine some genericity result in the spirit of [20], [21], [22] which could, for example, be stated like:

Open problem 2: Let $\Omega$ be an open set such that $\varphi_{1}^{2}, \varphi_{2}^{2}, \ldots, \varphi_{N}^{2}$ are linearly dependent. Then, prove that there exists arbitrary small deformations of its boundary such that the square of the eigenfunctions of the perturbed domain become linearly independent.

Following the step 5 of the proof of Theorem 3.1, if (5.1) is true (and therefore a unique optimal domain exists), this optimal domain can also be described as a level set of some linear combination of $\varphi_{1}^{2}, \varphi_{2}^{2}, \ldots, \varphi_{N}^{2}$. Now, the other results of this paper, Theorems 3.2 and 4.1 seem more difficult to prove in the two-dimensional case, even if the authors believe that they are true.

\section{REFERENCES}

[1] A. Auslender, Optimisation : Méthodes numériques, Paris/New York/Barcelone/Milan, Masson, 1976

[2] M.J. Balas, Active control of flexible systems, J. Optim. Theory Appl., volume(25), no. 3, 415-436, 1978

[3] C. Bardos, G. Lebeau and J. Rauch, Sharp sufficient conditions for the observation, control and stabilization of waves from the boundary, SIAM J. Control Optim., volume(30), pp. 1024- 1065, 1992.

[4] E. Bednarzuk, M. Pierre, E. Rouy and J. Sokolowski, Tangent sets in some functional spaces, Nonlinear Anal., volume(42), no 5, pp. 871-876, 2000.

[5] C. Castro and S. Cox, Achieving arbitrarily large decay in the damped wave equation, SIAM J. Control Optim., volume(39), no 6 , pp. 1748-1755, 2001.

[6] G. Chen, S.A. Fulling, F.J. Narcowich and S. Sun, Exponential decay of energy of evolution equations with locally distributed damping, SIAM J. Appl. Math., volume(51), no 1, pp. 266-301, 1991.

[7] R. Cominetti and J.P. Penot, Tangent sets to unilateral convex sets, C. R. Acad. Sci. Paris Sr. I Math, volume(321), no. 12, pp. 1631-1636, 1995.

[8] S. Cox and M. Overton, Perturbing the critically damped wave equation, SIAM J. Appl. Math., volume(56), no 5 , pp. 1353-1362, 1996.

[9] S. Cox and E. Zuazua, The rate at which energy decays in a damped string, Comm. in partial differential equations, volume(19), pp. 213-243, 1994.

[10] G. Dal Maso, An introduction to Г-convergence, Birkhäuser, Boston, 1993.

[11] P. Freitas, Optimizing the rate of decay of solutions of the wave equation using genetic algorithms: a counterexample to the constant damping conjecture, SIAM J. Control Optim., volume(37), no 2 , pp. 376-387, 1999.

[12] P. Freitas, On some eigenvalue problems related to the wave equation with indefinite damping, J. Differential Equations, volume(127), no 1, pp. 320-335, 1996.

[13] P. Freitas and E. Zuazua, Stability results for the wave equation with indefinite damping J. Diff. Equations, volume(132) (2), 338-352, 1996.

[14] P. Hébrard, PhD thesis, Université Henri Poincaré Nancy, http://www.iecn.u-nancy.fr/ hebrard/these_hebrard.ps.

[15] P. Hébrard and A. Henrot, Optimal shape and position of the actuators for the stabilization of a string, Systems and Control Letters, volume(48), pp. 199-209, 2003.

[16] A. Henrot and M. Pierre, Variations et Optimisation de formes, to appear in Mathématiques 
et Applications, Springer.

[17] J.B. Hiriart-Urruty and C. Lemaréchal, Convex analysis and minimization algorithms, Berlin, Heidelberg, New York, Springer, 1993.

[18] G. Lebeau, Équation des ondes amorties, Algebraic and geometric methods in mathematical physics Math. Phys. Stud., 19, Kluwer Acad. Publ., Dordrecht, 1996, p 73-109.

[19] T.J. Mahar and B.E. Willner, Sturm-Liouville eigenvalue problems in which the squares of the eigenfunctions are linearly dependent Comm. Pure Appl. Math. volume(33), no. 4, pp. 567-578, 1980.

[20] A.M. Micheletti, Perturbazione dello spettro dell'operatore di Laplace, in relazione ad una variazione del campo, Ann. Scuola Norm. Sup. Pisa, volume (3) 26, 151-169, 1972.

[21] J. Ortega and E. Zuazua, Generic simplicity of the spectrum and stabilization for a plate model, SIAM J. Cont. Optim., volume(39) (5), 1585-1614, 2001. (Addendum: 42 (5) (2004), 1905-1910).

[22] J. Ortega and E. Zuazua, On a constrained controllability problem for the heat equation, J. Optim. Theory. Appls., volume(108) (1), 29-64, 2001. (Addendum: 118 (1) (2003), 183-190.).

[23] L.R. Tcheugoue and E. Zuazua, Uniform exponential long time decay for the space semidiscretizations of a damped wave equation with artificial numerical viscosity, Numerische Mathematik, volume 95, no 3, p. 563-598, 2003.

[24] L.R. Tcheugoue and E. Zuazua, Uniform boundary stabilization of the finite difference space discretization of the $1-d$ wave equation, Advances in Computational Mathematics, to appear.

[25] L.N. Trefethen, Group velocity in finite difference schemes SIAM Rev, volume(24), no. 2, 113-136, 1982.

[26] E. Zuazua, Propagation, observation, and control of waves approximated by finite difference methods SIAM Review, to appear. 